\title{
SoxS represses flagellar gene expression through RfIP in Salmonella enterica Serovar Typhimurium
}

3

4 Srinivas S. Thota ${ }^{1}$, Brittany N. Henry ${ }^{1}$, and Lon M. Chubiz ${ }^{1,2,{ }^{*}}$

5

$6 \quad{ }^{1}$ Department of Biology, University of Missouri - St. Louis, St. Louis, MO 63121

$7{ }^{2}$ Biochemistry and Biotechnology Program, University of Missouri - St. Louis, St. Louis, MO

$8 \quad 63121$

9

10 *Correspondence: Lon M. Chubiz, University of Missouri - St. Louis, 1 University Blvd., St.

11 Louis. MO 63121; chubizl@umsl.edu

12

13

Running title: SoxS repression of flagella through RfIP/YdiV

14

15 


\section{ABSTRACT}

17 Flagellar gene expression is subject to regulation by many global transcription factors in

18 response to environmental and nutritional signals. One of the primary ways this occurs in

19 Salmonella enterica serovar Typhimurium, and its close relatives, is through controlling levels of

$20 \mathrm{FlhD}_{4} \mathrm{C}_{2}$ (the flagellar master regulator) via transcriptional, post-transcriptional, and post-

21 translational mechanisms. Recently, we found the homologous transcription factors MarA, Rob,

22 and SoxS repress fIhDC expression by distinct mechanisms. MarA and Rob, regulators involved

23 in inducible multidrug resistance, repressed fIhDC transcription by interacting directly with the

$24 \mathrm{flhDC}$ promoter. Alternatively, SoxS, the oxidative stress response regulator, altered $\mathrm{FlhD}_{4} \mathrm{C}_{2}$

25 levels independent of $f / h D C$ transcription by post-transcriptional or post-translational

26 mechanism. Here, using a forward genetic screen, we discovered that SoxS-dependent

27 repression of flagellar gene expression occurs through $\mathrm{RfIP}$, an anti-FlhD $\mathrm{C}_{2}$ factor that targets

$28 \mathrm{FlhD}_{4} \mathrm{C}_{2}$ for proteolytic degradation. Elevated soxS expression resulted in concomitant

29 increases in rfIP expression, indicating SoxS may work through RflP at the level of $r f I P$

30 transcription. Mapping of the rfIP promoter and a bioinformatic search yielded a putative SoxS

31 binding site proximal to the rfIP transcription start site. Comparison of the rfIP promoter region in

32 S. Typhimurium and Escherichia coli indicate substantial differences, providing a possible

33 mechanism for differential expression of $r f l P$ between these species.

\section{IMPORTANCE}

36 Salmonella enterica is a major cause of foodborne illness. Understanding environmental and

37 intracellular signals used by Salmonella to control expression of virulence-associated traits is

38 critical to advancing treatment and prevention of Salmonella-related disease. Reduced

39 expression of flagella at key points during Salmonella infection aids in evasion of the host innate

40 immune system. Within macrophages Salmonella is non-flagellated and exposed to oxidative 
41 stress. SoxS-dependent repression of flagellar genes may provide a potential link between

42 oxidative stress and reductions in flagellar expression.

\section{INTRODUCTION}

45 Flagellar motility is a primary means of locomotion for many bacterial species. While this form of 46 motility allows migration to more favorable environments, the production and operation of

47 flagella are significant biosynthetic and energetic burdens for flagellated bacteria (1, 2). For this

48 reason, most bacteria tightly regulate the biosynthesis of flagella in response to diverse

49 environmental cues $(3,4)$.

In Salmonella enterica serovar Typhimurium, and related Enterobacteriaceae, flagellar

51 genes form a distinct regulon where expression is hierarchical $(3,5)$. In this arrangement,

52 flagellar genes are temporally expressed in three distinct classes that correlate to specific

53 stages of flagellar assembly. Atop the hierarchical flagellar regulon is the heterohexameric

54 transcription factor $\mathrm{FlhD} \mathrm{hC}_{2}$, the product of the Class I operon $f \mathrm{hDC}(6)$. $\mathrm{FlhD}_{4} \mathrm{C}_{2}$ is considered

55 the master regulator that activates expression of several Class II genes corresponding to

56 components of the flagellar basal body and includes an alternative sigma factor, FliA (or $\sigma^{28}$ ) $(7-$

57 9). FliA subsequently directs transcription of an array of Class III genes encoding the flagellar

58 motor and filament, as well as proteins involved in chemotaxis (10). Additionally, a number of

59 internal regulatory feedbacks exist to ensure sequential expression of Class II and Class III

60 genes including autorepression of $f / h D C$ expression by $\mathrm{FlhD}_{4} \mathrm{C}_{2}$, FliT-dependent inhibition of

$61 \mathrm{FlhD}_{4} \mathrm{C}_{2}$ activity, indirect activation of $\mathrm{FlhD}_{4} \mathrm{C}_{2}$ activity by FliZ, and FlgM (a secretion-dependent

62 anti-FliA factor) inactivation of FliA (11-18).

Apart from autogenous control, numerous environmental and nutritional signals regulate

64 flagellar gene expression. Integration of these signals, and the resulting regulation of flagellar

65 gene expression, occurs primarily through control of $f / h D C$ expression or by altering the stability

66 of $\mathrm{FlhD}_{4} \mathrm{C}_{2}$. At the level of $f / h D C$ transcription, several global transcription factors involved in 
67 nutrient and envelope stress sensing serve as activators and repressors including: CRP, Fis,

68 Fur, H-NS, RcsB, and OmpR; among several others (11, 19-24). Additionally, the homologous

69 multidrug resistance-associated transcription factors MarA and Rob were recently found to

70 directly repress flhDC transcription in S. Typhimurium (25). Beyond transcription-level control, a

71 number of small regulatory RNAs (sRNA) are known to control flhDC expression at the mRNA

72 level (26). Together, these mechanisms carefully titrate the concencentration of $\mathrm{FlhD}_{4} \mathrm{C}_{2}$

73 produced across variable environments and growth stages.

74 While transcriptional and post-transcriptional mechanisms control $\mathrm{FlhD}_{4} \mathrm{C}_{2}$ production,

75 protein-protein interactions between RfIP (also known as YdiV) and $\mathrm{FlhD}_{4} \mathrm{C}_{2}$ are known to

76 regulate $\mathrm{FlhD}_{4} \mathrm{C}_{2}$ levels by targeting $\mathrm{FlhD}_{4} \mathrm{C}_{2}$ for degradation by ClpXP protease (27). The

77 effects of RfIP on $\mathrm{FlhD}_{4} \mathrm{C}_{2}$ levels are strongly nutrient dependent (28-30). Despite being an

78 EAL-domain containing protein, RfIP has not been demonstrated to have dicyclic-GMP

79 phosphodiesterase activity $(28,31,32)$. Thus, RfIP regulation of $\mathrm{FlhD}_{4} \mathrm{C}_{2}$ abundance occurs

80 through changes in RfIP concentrations dictated by rfIP transcription (15, 28-30, 33). Detailed

81 mapping of $r f I P$ regulation remains cryptic (15). However, nutrient and envelope stress have

82 been observed to increase rfIP expression $(28,34)$. Interestingly, despite being conserved in

83 flagellated Enterobacteriaceae, the effects of RfIP appear to be variable across and within

84 species (29). For example, RflP strongly regulates flagellar expression in S. Typhimurium and

85 pathogenic E. coli strains, but not in the laboratory model E. coli K-12 $(29,35)$. The variability in

86 RfIP usage is likely a result of differences in $r f l P$ regulation, but precise mechanisms remain

87 largely unexplored.

The superoxide stress response regulator SoxS is a potent repressor of flagellar gene

89 expression (25). Similar to its homologs MarA and Rob, SoxS is an AraC-family transcription

90 factor (36). Levels of SoxS are controlled through soxS transcriptional activation by the redox-

91 sensing transcription factor SoxR and proteolytic degradation of SoxS by Lon protease (37-39).

92 SoxS is responsible for directly and indirectly activating and repressing over 30 genes in E. coli 
$93(40,41)$. Many of the genes regulated by SoxS code for proteins involved in detoxifying

94 intracellular superoxide, efflux of toxic compounds, decreasing membrane permeability, and

95 altering central carbon metabolism $(40,42)$. More recently, SoxS was observed to repress

96 expression of the flagellar regulon S. Typhimurium (25). SoxS-dependent flagellar repression

97 occurred through reductions in $f / h D C$ transcription and post-transcriptional repression of

$98 \mathrm{FlhD}_{4} \mathrm{C}_{2}$ production in an Hfq-independent manner. The identity of factors involved in SoxS-

99 dependent repression of flagellar gene expression are unknown.

$100 \quad$ Here, we have discovered SoxS represses flagellar gene expression by reducing

$101 \mathrm{FlhD}_{4} \mathrm{C}_{2}$ concentrations via increased expression of $r f l P$. Using a transposon-based forward

102 genetic screen, we identified several independent insertion mutants in the rfIP coding and

103 promoter regions. Disruption of rflP obviated SoxS-dependent repression of Class II and Class

104 III gene expression, restoring motility. The regulatory connection between SoxS and rfIP

105 appears to be unique in Salmonella. The ability of SoxS to increase rfIP expression and reduce

106 flagellar gene expression may have a broader role in the virulence life cycle of Salmonella

107 where flagellation is regulated in response to conditions within the host, particularly the

108 presence of strong oxidizing agents.

\section{RESULTS}

\section{SoxS-dependent repression of flhDC expression occurs primarily through a post-}

112 transcriptional mechanism.

We have previously shown SoxS represses all three classes of flagellar genes (25).

114 Similar effects on flagellar gene expression were observed for the SoxS homologs MarA, Rob,

115 and RamA. Specifically, when SoxS was overproduced we found it repressed flhDC expression

116 at the transcriptional and post-transcriptional levels (25). To better understand the effects of

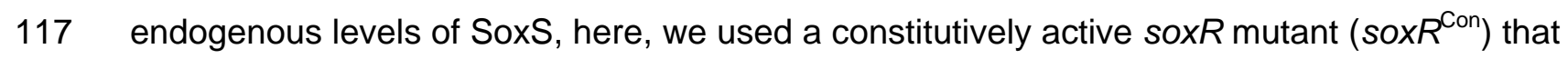

118 expresses soxS at roughly $50 \%$ of its maximally induced levels $(25,43,44)$. A constitutive 
119 mutant was utilized to avoid any possible pleiotropic effects of SoxR activating compounds on

120 flagellar gene expression. Using the sox $R^{\mathrm{Con}}$ mutant, we measured the effects of SoxS on

121 flagellar gene transcription, levels of FlhC and FliC proteins, and motility (Figure 1).

We measured transcription of $f l h D C, f l h B$ and fliC as representatives of Class I, Class II

123 and Class III flagellar genes, respectively, in sox $R^{\mathrm{Con}}$ and sox $R^{\mathrm{WT}}$ backgrounds (Figure 1A).

124 Transcription from each promoter was measured using chromosomally-integrated promoter

125 fusions to $y f p($ Venus) similar to Koirala and coworkers (45). We found transcription of flhB and

126 fliC were reduced to $15.9 \pm 2.9 \%$ (Student's $t$-test, $P=1.1 \times 10^{-8}$ ) and $5.9 \pm 1.6 \%$ (Student's $t$-test,

$127 P=1.6 \times 10^{-7}$ ) in the sox $R^{\mathrm{Con}}$ genetic background compared to sox $R^{\mathrm{WT}}$. Interestingly, there was no

128 significant change in the transcription of $f / h D C$ under the same conditions. These results are

consistent with SoxS activating a post-transcriptional mechanism to repress $f / h D C$ expression.

Reductions in Class II and Class III transcription were reflected in decreased levels of

131 FliC protein and motility in the sox $R^{\mathrm{Con}}$ background compared to sox $R^{\mathrm{WT}}$. Levels of FliC were

132 qualitatively reduced below detectable limits in the sox $R^{\text {Con }}$ background (Figure 1B). We also

133 observed qualitative reductions in levels of FlhC protein in the sox $R^{\text {Con }}$ background compared to

134 sox $R^{\mathrm{WT}}$, providing a likely reason for reductions in FliC (Figure 1B). Similarly, swimming

135 motility of the sox $R^{\text {Con }}$ mutant was reduced to $66.3 \pm 5.0 \%$ of wild-type (Figure 1C; Student's $t$ -

136 test, $P=3.5 \times 10^{-12}$ ). Taken together, the absence of changes in flhDC transcription and

137 decreased levels of FlhC protein levels in response to endogenous levels of SoxS suggest

138 SoxS represses fIhDC expression at the post-transcriptional or post-translational levels.

\section{Identification of mutants that bypass SoxS-dependent flagellar repression}

We employed a transposon-based forward genetic screen in the sox $R^{\mathrm{Con}}$ background for

142 mutants with recovered flagellar gene expression. Previously, we demonstrated in vivo

143 interactions between SoxS and the fIhDC promoter are weak and may have limited biological

144 significance (25). Here, we showed constitutive expression of SoxS at endogenous levels 
represses fIhDC post-transcriptionally (Figure 1). These lines of evidence point to SoxS regulating $f / h D C$ expression indirectly through another pathway. To identify pathways involved in

147 SoxS-dependent flagellar repression we performed a forward genetic screen in our sox $R^{\text {Con }}$

148 background using the T-POP transposon, similar to the approach used by Wozniak and

149 coworkers to identify flagellar regulators (46). In total, $>100,000$ mutants from 6 independent

150 pools were screened for restoration of fliC transcription. The unique mutants recovered with

151 restored fliC expression were 6 insertions in $r f I P$ (an anti-FlhD ${ }_{4} \mathrm{C}_{2}$ factor) (Figure 2A), 3

152 insertions in fliD (the flagellar filament cap protein), 2 insertions in $\operatorname{rcs} B$ (the Rcs response

153 regulator); and 1 insertion each in topA (topoisomerase I), $\operatorname{prgH}$ (a SPI1 type III secretion

154 structural protein), and fliC (the flagellar filament protein), respectively. Remarkably, we

155 observed no T-POP insertions in the soxRS locus. However, the extensive T-POP screen by

156 Wozniak and coworkers did not uncover soxS as a flagellar regulator, as well; suggesting the

157 soxRS locus may be a cold spot for the T-POP transposon system (46).

The recovered T-POP insertion mutations fell into two major categories. First were

159 flagellar genes with possible autogenous regulatory effects on flagellar gene expression. For

example, insertions in fliD likely have polar effects on fliT, a gene distal to fliD in the same Class

161 II operon (fliDST). FliT is a chaperone for several flagellar structural proteins and also serves as

162 a negative regulator of $\mathrm{FlhD}_{4} \mathrm{C}_{2}$ activity via protein-protein interactions (13). Loss of fliT is known

163 to increase expression of Class II and Class III genes $(12,13)$. Thus, removal of a FlhD ${ }_{4} \mathrm{C}_{2}$

164 inhibitor may increase expression of fliC. Since SoxS is likely utilizing a pathway external to the

165 flagellar regulon, we did not prioritize further analysis of fliD insertions. Second were genes with

166 autonomous roles in flagellar gene regulation. Specifically, rfIP and rcsB have known

167 interactions with $\mathrm{FlhD}_{4} \mathrm{C}_{2}$ and the flhDC promoter, respectively. These negative interactions

168 reduce Class II activation $(23,27,28,47)$. From the second category, we looked to examine the 169 effects of rfIP as its effects on $\mathrm{FlhD}_{4} \mathrm{C}_{2}$ are post-translational, consistent with our observations 170 surrounding SoxS-dependent repression of Class I expression (27). 
Inactivation of rfIP alleviates SoxS-dependent repression of flagellar gene expression and motility

We tested whether SoxS repressed flagellar genes through RfIP by observing the effects of a rfIP mutant on flagellar gene expression, levels of FlhC protein, and motility in the sox $R^{\text {Con }}$ genetic background. Similar to previous observations, the expression of flhB (Class II) and fliC

177 (Class III) flagellar genes were reduced to $12.9 \pm 4.2 \%$ (Student's $t$-test, $P=1.5 \times 10^{-9}$ ) and

$1787.0 \pm 1.4 \%$ (Student's $t$-test, $P=9.6 \times 10^{-9}$ ) of the wild-type levels, respectively, in the sox $R^{\text {Con }}$ strain

179 (Figure 2B). Inactivation of $r f I P$ resulted in rescue of $f / h B(91.7 \pm 7.7 \%$, Student's $t$-test, $P=0.14)$

180 and fliC $\left(89.3 \pm 2.5 \%\right.$, Student's $t$-test, $\left.P=2.0 \times 10^{-3}\right)$ expression to nearly wild-type levels in the $r f I P$

181 sox $R^{\text {Con }}$ background strain (Figure 2B). Likewise, the levels of FlhC protein were reduced in

182 sox $R^{\mathrm{Con}}$, as expected, but were qualitatively recovered in the $\operatorname{rfl}$ sox $R^{\mathrm{Con}}$ genetic background

183 (Figure 2C). Notably, the levels of FlhC in the rfIP sox $R^{\text {Con }}$ background, while increased

184 compared to sox $R^{\text {Con }}$, remain qualitatively lower than wild-type FlhC levels. This might be due to

185 the presence of an additional SoxS-dependent pathway affecting $\mathrm{FlhD}_{4} \mathrm{C}_{2}$ stability, or the

186 elevated levels of SoxS expression may indirectly increase $\mathrm{FlhD}_{4} \mathrm{C}_{2}$ degradation rates.

187 Consistent with recovery of Class II/III gene expression and FlhC levels, the $38.4 \pm 3.7 \%$

188 (Student's $t$-test, $P=2.2 \times 10^{-6}$ ) reduction in swimming motility in the sox $R^{\text {Con }}$ background was 189 recovered to near wild-type levels in the $\operatorname{rfIP}$ sox $R^{\mathrm{Con}}$ background (Figure 2D).

We also wanted to determine if SoxS-dependent repression of flagellar genes can be

191 complemented in the $r f I P$ sox $R^{\text {Con }}$ strain when $r f I P$ is provided in trans. The rflP locus, including

192 the native rfIP promoter (described below), was introduced into the sox $R^{\mathrm{Con}}$ and $r f I P$ sox $R^{\mathrm{Con}}$

193 background on a low-copy number plasmid (pRfIP). When harboring pRfIP, the sox $R^{\mathrm{Con}}$ and $r f I P$

194 sox $R^{\text {Con }}$ backgrounds expressed fliC at $8.5 \pm 0.6 \%$ (Student's $t$-test, $P=6.1 \times 10^{-6}$ ) and $13.3 \pm 0.9 \%$

195 (Student's $t$-test, $P=9.4 \times 10^{-6}$ ) of levels observed in the sox $R^{\text {Con }}$ strain harboring the empty

196 vector (pWKS30) (Figure 3A). Consistent with SoxS working through rfIP, the level of fliC 
197 expression in the $r f I P$ sox $R^{\text {Con }}$ background is $15.2 \pm 2.2$ fold (Student's $t$-test, $P=2.4 \times 10^{-6}$ ) more

198 than that of $\operatorname{sox} R^{\mathrm{Con}}$ strain bearing the control vector. Motility was also repressed in sox $R^{\text {Con }}$ and 199 rfIP sox $R^{\text {Con }}$ background carrying pRfIP compared to same strains harboring the empty vector

200 (Figure 3B). Overall, these data suggest SoxS works through RfIP to decrease levels of

$201 \quad \mathrm{FlhD}_{4} \mathrm{C}_{2}$. The reduction in $\mathrm{FlhD}_{4} \mathrm{C}_{2}$ levels is a likely explanation for reduced expression of Class

202 II and Class III genes, ultimately manifesting in reduced motility.

203

204 Increased soxS expression results in elevated rfIP promoter activity.

We looked to see what effects increased soxS expression had on rfIP promoter activity.

206 One possible mechanism for SoxS to reduce flagellar gene expression through RfIP is via

207 increasing rflP transcription. Notably, the dosage of rfIP expression is believed to control RflP-

208 dependent reductions in levels of $\mathrm{FlhD}_{4} \mathrm{C}_{2}$ and not activation by small molecule signals, as the

209 EAL domain of RfIP is non-functional $(28,32,33)$. Based on known properties of RfIP-FlhD ${ }_{4} \mathrm{C}_{2}$

210 interactions and genetic evidence that SoxS functions through $r f I P$, we hypothesized elevated

211 levels of soxS expression may yield corresponding increases in rflP expression. To test this

212 hypothesis, we used an in situ lacZY promoter fusion to rfIP in sox $R^{\mathrm{Con}}$ and sox $R^{\mathrm{WT}}$ background

213 strains. We found that $r f I P$ expression was $2.4 \pm 0.1$ fold (Student's $t$-test, $P=4.8 \times 10^{-6}$ ) higher in

214 sox $R^{\text {Con }}$ compared to wild-type (Figure 4A). One interpretation may be that SoxS is interacting

215 with the rflP promoter and serving as an activator. The lack of transposon mutants in genes

216 coding for transcription factors regulated by SoxS further supports this possibility.

217 Notwithstanding, the paucity of information on rflP promoter structure limits any further

218 speculation.

219

220 Mapping of the rfIP promoter.

221 To better understand the possible interaction between SoxS and the rflP promoter, we 222 mapped the rflP transcriptional start site (TSS). Previous mapping of the rfIP promoter has been 
223 via bioinformatic approaches $(48,49)$. Using these predictions, we found a cloned rfIP promoter

224 fusion cloned in trans displayed no response to known nutritional cues or SoxS levels (data not

225 shown). We interpreted these data to mean that the native rfIP promoter location may have

226 been incorrectly mapped or may be more complex than previously predicted, as suggested by

227 Wada and colleagues (29). Using template-switching rapid amplification of cDNA ends (5'-

228 RACE) to sequence the 5'-end of the rfIP mRNA, we identified the transcription start site of rfIP

229 to $-230 \mathrm{bp}$ of $r f \mathrm{P}$ start codon (Figure 4B). This is over $150 \mathrm{bp}$ upstream of previous predictions

230 in $E$. coli and very close to $n / p C$, the gene just proximal to $r f I P$ (Figure 2A) $(48,49)$. Consistent

231 with the location of the promoter identified, in our transposon screen we only observed

232 insertions in the $r f I P$ coding region or the nIpC-rfIP intergenic region distal to the $r f I P$ TSS, and

233 not in the nlpC gene (Figure 2A). This is a markedly different result than other studies in

234 Salmonella that have noted disruptions in $n / p C$ have polar effects on rfIP expression (15, 29,

235 46). An explanation for this difference may be that SoxS utilizes the native rfIP promoter to 236 increase rfIP expression while other regulators, such as FliZ, utilize the proximal $n / p C$ promoter

237 to mediate changes in rfIP expression (15). Taken together, these data show regulation of rfIP is 238 complex and may involve multiple promoters.

Identification of a putative SoxS binding site in the rfIP promoter.

Since rflP transcription was elevated when SoxS expression was increased, we wanted

242 to bioinformatically search for any possible SoxS binding sites (soxbox) in the rflP promoter

243 region. To locate possible SoxS binding sites in the rfIP promoter, we employed the MEME motif

244 search algorithm using 29 known and putative SoxS binding sites in E. coli promoters as a

245 training data set (50). The rfIP promoters from $S$. Typhimurium and E. coli were added to detect

246 possible SoxS binding motifs. E. coli sequences were chosen due to the paucity of well-

247 characterized SoxS binding sites in S. Typhimurium, although many of genes are similarly

248 regulated by SoxS (51). Using these known soxbox containing promoters, MEME identified a 
conserved soxbox motif in the $S$. Typhimurium rfIP (Figure 4B). The putative SoxS binding site (5'TGGCAGGCACGGCGAA3') located 88 bp upstream of the rfIP TSS is similar to soxbox motifs from studies by Seo and coworkers (5'-AYRGCAYWAWWTRYYAAW-3') and Martin and workers (5'-AYNGCACNNWNNRYYAAA-3') $(41,52)$. While the predicted SoxS binding site is

253 further upstream from the TSS than soxbox sites in many well-studied promoters (52); examples

254 such as the micF promoter indicate SoxS binding sites can be even further upstream of the TSS

255 (i.e. >100 bp) (53). Future biochemical mapping of SoxS interactions with the S. Typhimurium

256 rfIP promoter will help validate these predictions.

Interestingly, this same site was predicted in the E. coli rfIP promoter. Notably, the

258 putative $S$. Typhimurium and $E$. coli SoxS binding sites vary in the region between the

259 conserved 5'-GCA-3' and 5'-YYAA-3' minor motifs (Figure 4B). These differences may provide

260 an explanation as to why SoxS has not been found to bind to the rfIP promoter or regulate $r f I P$

261 transcription in E. coli (41).

The rfIP promoter regions of S. Typhimurium and E. coli have limited similarity

Having mapped a rfIP promoter region by 5'-RACE in S. Typhimurium, we looked to compare this region to the corresponding chromosomal region in E. coli $\mathrm{K}-12$ to determine if the rfIP promoter was conserved. Comparison of the rfIP promoter region in S. Typhimurium to the corresponding region in E. coli indicated substantial differences (Figure 4B). In particular, the region corresponding to $S$. Typhimurium rfIP TSS bears little similarity to the same region in $E$. coli (41.3\% identity between the rfIP TSS and putative soxbox). This result indicates the rfIP

270 promoter structure may be drastically different in E. coli as compared to S. Typhimurium. In E.

271 coli, genome-scale transcriptomics and chromatin immunoprecipitation sequencing (ChIP-seq)

272 experiments involving SoxS have not identified $r f I P$ as a SoxS target (41). The difference in $r f I P$ 273 promoter sequences may explain the absence of SoxS interactions with rfIP in E. coli. 


\section{DISCUSSION}

We have demonstrated that SoxS represses flagellar gene expression primarily through

277 elevating levels of rfIP (also known as ydiV) expression. In a recent study, we established the

278 intracellular superoxide response regulator SoxS is a repressor of flagellar genes primarily

279 through reductions in flhDC (Class I) expression (25). While the homologs of SoxS, specifically

280 MarA and Rob, were found to repress $f / h D C$ by direct interaction with the flhDC promoter, SoxS

281 was found to decrease $\mathrm{FlhD}_{4} \mathrm{C}_{2}$ levels independent of effects on flhDC transcription (25). Post-

282 transcriptional regulation of $f / h D C$ expression by SoxS was found to be independent of Hfq, the

283 sRNA chaperone (25). This largely discounted the effects of sRNA as a possible mechanism as

284 most sRNA that regulate fIhDC mRNA stability and translation are known to require Hfq (54). To

285 elucidate factors involved in SoxS-dependent post-transcriptional repression of flhDC, we

286 performed a transposon-based forward genetic screen for mutants with recovered flagellar gene

287 expression in a genetic background where SoxS is constitutively produced. Several

288 independent insertion mutants mapped to $r f I P$. Subsequent genetic analysis confirmed that $r f I P$

289 is indeed the link between increased SoxS levels and repression of flagellar gene expression.

290 Mapping of the rfIP transcription start site and resulting bioinformatic analysis identified a

291 putative soxbox in the Salmonella rfIP promoter region. These results shed light on a novel

292 regulatory pathway connecting oxidative stress and flagellar gene regulation.

SoxS-dependent activation of rflP occurs in S. Typhimurium but not E. coli K-12 (41).

294 Our bioinformatic analysis identified a putative soxbox in the rfIP promoter in S. Typhimurium.

295 The corresponding site was also present in E. coli; however, E. coli lacks the promoter region

296 identified in S. Typhimurium. Transcriptomic and DNA binding studies of SoxS have indicated

297 several common targets between E. coli and Salmonella, however, these do not include rflP (41,

$29851,55)$. The strikingly different sequences of the rflP promoter region between these two

299 species may provide a possible mechanism for the lack of SoxS-dependent activation of rfIP in

300 E. coli (Figure 4B). Further comparative studies between the SoxS regulons of these species 
301 will provide a better picture of how each species differentially uses the response to oxidative

302 stress to alter physiology and metabolism, as demonstrated by SoxS regulation of flagellar 303 expression.

304 More broadly, control of $r f I P$ may be an important aspect of flagellar expression in 305 response to intracellular chemical stress in the Enterobacteriaceae. In species where RfIP is a 306 prominent mechanism for flagellar regulation, levels of $r f I P$ expression have been found to be 307 responsive to nutritional status, envelope stress, and the cell's physiochemical environment (28, $30834,35)$. Adding to the complexity of $r f I P$ regulation is the action of SoxS, a key mediator of the 309 oxidative stress response in Salmonella. These examples highlight how rfIP has been integrated 310 into diverse stress responses of this bacterial family. While this study did not directly explore the 311 interactions between SoxS and the rfIP promoter, future studies examining the role of SoxS-

312 dependent regulation of rfIP throughout the Enterobacteriaceae will more clearly define this

313 interaction and provide deeper insights into adaptive benefits of reducing flagellar expression in 314 response to oxidative stress.

315 The relationship between SoxS and rflP expression could have an impact on Salmonella 316 virulence. Notably, RflP-dependent reductions in flagellar expression aid in evasion of the host 317 caspase-1 inflammatory response, enhancing the ability of Salmonella to survive during 318 systemic infection (56). During this process, Salmonella is exposed to oxidative stress which is 319 known to be a chemical cue for expression of certain virulence traits. The role of SoxS in this 320 process is unclear. Earlier work by Fang and coworkers found SoxS is not involved in virulence 321 (57). More recently, Wang and coworkers have discovered that SoxS does indeed play a 322 regulatory role in Salmonella virulence (55). Specific causes for this discrepancy are unresolved 323 but may be due to differences in the soxS mutants used in each study (55). While it is tempting 324 to draw connections between SoxS, RflP, and Salmonella's survival within the host, more 325 detailed studies of the mechanism behind SoxS-dependent effects on Salmonella virulence are 326 needed. 
Regulation of stress responses and structural genes is complex and has diverged even

328 amongst closely related bacterial species such as $S$. Typhimurium and E. coli (58, 59). In $S$.

329 Typhimurium we have previously shown that the multidrug resistance regulators MarA, SoxS,

330 and Rob repress flagellar gene expression in contrast to what has been observed in E. coli (25).

331 Building on this, we have shown here that even amongst homologous transcription factors there

332 exists different mechanisms to achieve the same ends. SoxS-dependent repression of flagellar

333 expression through RfIP is so far unique to $S$. Typhimurium. Further exploration of how RfIP is

334 used in flagellar regulation in other motile Enterobacteriaceae may shed light on how the costs

335 and benefits of flagellar motility are managed in response to the complex environment inhabited

336 by this bacterial family.

\section{MATERIALS AND METHODS}

\section{Bacterial strains, plasmids and media.}

340 All the bacterial strains and plasmids generated in this study are listed in Table S1. For all

341 experiments, cultures were propagated in tryptone broth ( $1 \%$ tryptone, $0.8 \% \mathrm{NaCl})$ and grown at

$34237^{\circ} \mathrm{C}$. Swimming motility assays were performed in soft agar ( $0.3 \%$ Bacto agar, $1 \%$ tryptone, and

$3430.8 \% \mathrm{NaCl}$ ). All genetic manipulations were conducted with cells grown in Luria-Bertani liquid

344 and solid media ( $1 \%$ tryptone, $0.5 \%$ yeast extract, $1 \% \mathrm{NaCl}$, and $1.6 \%$ Bacto agar for solid

345 media). Bacterial cultures were grown at $37^{\circ} \mathrm{C}$ for all experiments or $30^{\circ} \mathrm{C}$ for strains carrying

346 temperature-sensitive plasmids, unless otherwise specified. Where required, media was

347 supplemented with kanamycin, carbenicillin, chloramphenicol, or tetracycline at a final

348 concentration of $50 \mu \mathrm{g} / \mathrm{ml}, 100 \mu \mathrm{g} / \mathrm{ml}, 30 \mu \mathrm{g} / \mathrm{ml}$, or $15 \mu \mathrm{g} / \mathrm{ml}$, respectively.

\section{Genetic manipulations.}

351 All strains used in study are derivatives of Salmonella enterica serovar Typhimurium LT2. The

352 sequences of oligonucleotides (IDT) used in genetic manipulation of strains are provided in 
353 Table S2. Gene knockouts were created using the $\lambda$ Red method of Datsenko and Wanner using

354 the pKD46 recombinase helper plasmid and site-specific PCR fragments with homologous

355 overhangs generated using pKD13 or pKD32 plasmids as templates (60). Deletion mutants

356 were selected for on LB-kanamycin or LB-chloramphenicol agar plates. All deletion mutants

357 were backcrossed into relevant parental strains using P22 HT/int generalized transduction (61).

358 When necessary, antibiotic markers between the FRT sites were excised from mutants by

359 expressing Flp recombinase from pCP20 plasmid (60).

$360 \quad$ Transcriptional fusions were produced either in trans at the $\lambda$ attachment site or in situ.

361 All transcriptional fusions made in trans have been described in a previous study and used the

362 pVenus plasmid to produce $y f p$ transcription fusions $(25,62)$. The in situ transcriptional fusion to

363 rfIP used the FLP recombinase-mediated lacZY fusion system of Ellermeier and coworkers (63).

\section{Transposon mutagenesis}

366 Transposon mutagenesis was performed using the T-POP (Tn 10dTc[del-25]) transposon as

367 described by Lee and coworkers with minor modifications $(46,64,65)$. Briefly, high frequency T-

368 POP donor MudP22 lysates were generated with TH3923 and used to transduce T-POP into

369 LCM2473/pNK2881 cells. Resulting primary T-POP insertion mutants $(1,000-2,000$

370 colonies/plate) selected on LB-tetracycline agar were pooled, treated with P22 HT int-105. In

371 total, 6 independent pools were generated. Random T-POP insertions from each pool were

372 transduced into LCM2473 followed by screening on LB-tetracycline agar plates. Colonies were

373 visually screened for restored fliC'-yfp expression using a $400 \mathrm{~nm}$ wavelength transilluminator

374 (IORodeo). A minimum of 20,000 T-POP mutants were screened per pool. Positive insertions

375 were mapped by degenerate PCR using primer P-LCM593 to P-LCM598 (Table S2) as

376 described by Lee and coworkers (65).

\section{Transcriptional reporter assays}


379 Transcriptional reporter assays measured either YFP fluorescence or $\beta$-galactosidase activity.

380 For YFP fluorescence and $\beta$-galactosidase activity assays, cultures were subcultured 1:1000

381 from overnight cultures and grown to mid-log phase $\left(\mathrm{OD}_{600} 0.6-0.8\right)$ prior to measurements. To

382 measure YFP fluorescence, $200 \mu \mathrm{L}$ of each culture was transferred to 96 -well black and clear

383 bottom plates (Corning Costar) and fluorescence was measured with a Cytation3 multimode

384 microplate reader (BioTek). Fluorescence was measured at wavelengths $500 \pm 5 \mathrm{~nm}$ excitation

385 and $525 \pm 5 \mathrm{~nm}$ emission and normalized to corresponding $\mathrm{OD}_{600}$ values. Each sample had a

386 minimum of 6 replicates.

387 The method of Slauch and Silhavy was used for $\beta$-galactosidase activity assays in 388 microtiter plates, with minor modifications (66). Samples (1 mL) from mid-log phase cultures 389 were pelleted at $6,000 \mathrm{xg}$ and resuspended in $\mathrm{Z}$ buffer $(0.1 \mathrm{M}$ sodium phosphate, $0.01 \mathrm{M} \mathrm{KCl}$,

$390 \quad 0.001 \mathrm{M} \mathrm{MgSO}_{4}, 0.05 \mathrm{M} \beta$-mercaptoethanol, $\mathrm{pH}$ 7.0). Two $100 \mu \mathrm{L}$ aliquots were saved to

391 measure $\mathrm{OD}_{600}$ prior to sample permeabilization. Samples were permeabilized via vortexing with

$39250 \mu \mathrm{L} 0.1 \%$ sodium dodecyl sulfate (SDS) and $100 \mu \mathrm{L}$ chloroform for $1 \mathrm{~min}$. From each sample,

393 two $100 \mu \mathrm{L}$ aliquots of permeabilized cell extract was added to the wells of a 96 well assay

394 plate. To initiate assays, $20 \mu \mathrm{L}$ of $o$-nitrophenyl- $\beta$-D-galactoside (ONPG) reagent $(20 \mathrm{mg} / \mathrm{ml}$ in $395 \quad Z$ buffer) was added followed by mixing at $600 \mathrm{rpm}$ on a microplate shaker (Thermo Scientific).

396 The change in absorbance at $420 \mathrm{~nm}$ was measured every $5 \mathrm{~min}$ for $1 \mathrm{hr}$ in a Cytation3

397 multimode plate reader (BioTek). The relative Vmax for each reaction was calculated using

398 Gen5 software (BioTek). $\beta$-galactosidase activity of cultures calculated as: $\operatorname{Vmax} /\left(\mathrm{OD}_{600}{ }^{*} 0.1\right.$

$399 \mathrm{~mL}$ ). Each sample had a minimum of 4 replicates. All data analysis and plotting were performed 400 in R.

401

\section{Motility Assays}

403 All motility assays used cultures grown to mid-log phase $\left(\mathrm{OD}_{600} 0.6-0.8\right)$ that were normalized

404 to the lowest measured cell density. For each assay, $1 \mu \mathrm{L}$ of culture was inoculated into motility 
405 agar plates and incubated for 6 hours at $37^{\circ} \mathrm{C}$. Images were obtained with a high-resolution

406 flatbed scanner (Epson) and were processed using ImageJ.

\section{Immunoblots}

409 Immunoblots for FliC, FlhC-3xFLAG, and DnaK were performed with $100 \mu \mathrm{g}$ of total protein

410 harvested from mid-log phase $\left(\mathrm{OD}_{600} 0.6-0.8\right)$ cultures per the procedure described elsewhere

411 (25).

\section{Transcription start site mapping}

414 A template-switching 5' rapid amplification of cDNA ends (5'-RACE) kit (New England BioLabs)

415 was used to map the transcription start site of $r f I P$. Total RNA extracted from mid-log phase

416 cells using TRIzol reagent (Life Technologies) and Direct-zol RNA recovery kits (Zymo

417 Research). cDNA was prepared from total RNA using the rfIP specific primer (P-LCM621) and

418 template-switching oligonucleotide (P-LCM619) using RT Template Switching Enzyme Mix

419 (New England Biolabs). The 5'-end end of the rfIP cDNA was enriched by PCR using the

420 primers P-LCM620 and P-LCM622. The enriched fragment is gel purified and sequenced

421 (Eurofins). Sequencing data was mapped to the S. Typhimurium LT2 genome using the Artemis 422 software suite.

\section{Bioinformatic analysis}

425 SoxS binding sites were predicted using the multiple EM for motif elicitation (MEME) algorithm

426 (50). A set of 29 E. coli promoters with annotated SoxS binding sites were used to guide the

427 detection of a SoxS binding motif in the rfIP promoters of E. coli and S. Typhimurium (Table

428 S3). The number of motifs the tool was allowed was set to 1 , and the minimum and maximum 429 width of the motif size allowed was set at $12 \mathrm{bp}$ and $30 \mathrm{bp}$, respectively. The rfIP promoter 
430 regions of $S$. Typhimurium, and its corresponding region in E. coli, were compared using

431 Multiple Sequence Comparison by Log- Expectation tool (MUSCLE) (67).

432

433 ACKNOWLEDGEMENTS

434 We thank C.M. Cenzer for technical assistance in transposon screening and mapping. This 435 work was supported by NIH grant Al137984 to L.M.C. 


\section{REFERENCES}

437 1. Mitchell JG. 2002. The energetics and scaling of search strategies in bacteria. Am Nat $438 \quad 160: 727-740$.

439 2. Macnab RM. 2003. How bacteria assemble flagella. Annu Rev Microbiol 57:77-100.

440 3. Chevance FFV, Hughes KT. 2008. Coordinating assembly of a bacterial macromolecular 441 machine. Nat Rev Microbiol 6:455-465.

442 4. Mukherjee S, Kearns DB. 2014. The structure and regulation of flagella in Bacillus subtilis. $443 \quad$ Annu Rev Genet 48:319-340.

444 5. Chilcott GS, Hughes KT. 2000. Coupling of flagellar gene expression to flagellar assembly 445 in Salmonella enterica serovar typhimurium and Escherichia coli. Microbiol Mol Biol Rev $446 \quad 64: 694-708$.

447 6. Kutsukake K, Ohya Y, lino T. 1990. Transcriptional analysis of the flagellar regulon of $448 \quad$ Salmonella typhimurium. J Bacteriol 172:741-747.

449 7. Liu X, Matsumura P. 1994. The FlhD/FIhC complex, a transcriptional activator of the $450 \quad$ Escherichia coli flagellar class II operons. J Bacteriol 176:7345-7351.

451 8. Mytelka DS, Chamberlin MJ. 1996. Escherichia coli fliAZY operon. J Bacteriol 178:24-34.

452 9. Ikebe T, lyoda S, Kutsukake K. 1999. Structure and expression of the fliA operon of 453 Salmonella typhimurium. Microbiology 145 ( Pt 6):1389-1396.

454 10. Ohnishi K, Kutsukake K, Suzuki H, lino T. 1990. Gene fliA encodes an alternative sigma 455 factor specific for flagellar operons in Salmonella typhimurium. Mol Gen Genet 221:139456 147. 
457 11. Kutsukake K. 1997. Autogenous and global control of the flagellar master operon, flhD, in 458 Salmonella typhimurium. Mol Gen Genet 254:440-448.

459 12. Kutsukake K, Ikebe T, Yamamoto S. 1999. Two novel regulatory genes, fliT and fliZ, in the $460 \quad$ flagellar regulon of Salmonella. Genes Genet Syst 74:287-292.

461 13. Yamamoto S, Kutsukake K. 2006. FliT acts as an anti-FlhD2C2 factor in the transcriptional control of the flagellar regulon in Salmonella enterica serovar Typhimurium. J Bacteriol

14. Saini S, Brown JD, Aldridge PD, Rao CV. 2008. FliZ Is a posttranslational activator of FlhD4C2-dependent flagellar gene expression. J Bacteriol 190:4979-4988.

15. Wada T, Tanabe Y, Kutsukake K. 2011. FliZ acts as a repressor of the ydiV gene, which encodes an anti-FlhD4C2 factor of the flagellar regulon in Salmonella enterica serovar Typhimurium. J Bacteriol 193:5191-5198.

16. Ohnishi K, Kutsukake K, Suzuki H, Lino T. 1992. A novel transcriptional regulation mechanism in the flagellar regulon of Salmonella typhimurium: an antisigma factor inhibits the activity of the flagellum-specific sigma factor, sigma F. Mol Microbiol 6:3149-3157.

17. Chadsey MS, Karlinsey JE, Hughes KT. 1998. The flagellar anti-sigma factor FlgM actively dissociates Salmonella typhimurium sigma28 RNA polymerase holoenzyme. Genes Dev

18. Chadsey MS, Hughes KT. 2001. A multipartite interaction between Salmonella transcription factor sigma28 and its anti-sigma factor FlgM: implications for sigma28 holoenzyme destabilization through stepwise binding. J Mol Biol 306:915-929.

478 19. Komeda Y, Suzuki H, Ishidsu JI, lino T. 1976. The role of cAMP in flagellation of 
Salmonella typhimurium. Mol Gen Genet 142:289-298.

480
20. Kelly A, Goldberg MD, Carroll RK, Danino V, Hinton JCD, Dorman CJ. 2004. A global role for Fis in the transcriptional control of metabolism and type III secretion in Salmonella enterica serovar Typhimurium. Microbiology 150:2037-2053.

21. Campoy S, Jara M, Busquets N, de Rozas AMP, Badiola I, Barbé J. 2002. Intracellular cyclic AMP concentration is decreased in Salmonella typhimurium fur mutants. Microbiology 148:1039-1048.

22. Bertin P, Terao E, Lee EH, Lejeune P, Colson C, Danchin A, Collatz E. 1994. The H-NS protein is involved in the biogenesis of flagella in Escherichia coli. J Bacteriol 176:55375540.

23. Wang Q, Zhao Y, McClelland M, Harshey RM. 2007. The RcsCDB signaling system and swarming motility in Salmonella enterica serovar Typhimurium: dual regulation of flagellar and SPI-2 virulence genes. J Bacteriol 189:8447-8457.

24. Shin S, Park C. 1995. Modulation of flagellar expression in Escherichia coli by acetyl phosphate and the osmoregulator OmpR. J Bacteriol 177:4696-4702.

25. Thota SS, Chubiz LM. 2019. Multidrug resistance regulators MarA, SoxS, Rob, and RamA repress flagellar gene expression and motility in Salmonella enterica serovar Typhimurium. J Bacteriol.

26. De Lay N, Gottesman S. 2012. A complex network of small non-coding RNAs regulate motility in Escherichia coli. Mol Microbiol 86:524-538.

27. Takaya A, Erhardt M, Karata K, Winterberg K, Yamamoto T, Hughes KT. 2012. YdiV: a dual function protein that targets FlhD4C2 for ClpXP-dependent degradation by promoting 
release of DNA-bound FlhD4C2 complex. Mol Microbiol 83:1268-1284.

502

503

504

505

506

507

508

509

510
28. Wada T, Morizane T, Abo T, Tominaga A, Inoue-Tanaka K, Kutsukake K. 2011. EAL domain protein YdiV acts as an anti-FlhD4C2 factor responsible for nutritional control of the flagellar regulon in Salmonella enterica serovar Typhimurium. J Bacteriol 193:1600-1611.

29. Wada T, Hatamoto Y, Kutsukake K. 2012. Functional and expressional analyses of the antiFlhD4C2 factor gene ydiV in Escherichia coli. Microbiology 158:1533-1542.

30. Koirala S, Mears P, Sim M, Golding I, Chemla YR, Aldridge PD, Rao CV. 2014. A nutrienttunable bistable switch controls motility in Salmonella enterica serovar Typhimurium. MBio 5:e01611-14.

31. Simm R, Remminghorst U, Ahmad I, Zakikhany K, Römling U. 2009. A role for the EAL-like protein STM1344 in regulation of CsgD expression and motility in Salmonella enterica serovar Typhimurium. J Bacteriol 191:3928-3937.

32. Hengge R, Galperin MY, Ghigo J-M, Gomelsky M, Green J, Hughes KT, Jenal U, Landini P. 2016. Systematic Nomenclature for GGDEF and EAL Domain-Containing Cyclic Di-GMP Turnover Proteins of Escherichia coli. J Bacteriol 198:7-11.

33. Li B, Li N, Wang F, Guo L, Huang Y, Liu X, Wei T, Zhu D, Liu C, Pan H, Xu S, Wang H-W, Gu L. 2012. Structural insight of a concentration-dependent mechanism by which YdiV inhibits Escherichia coli flagellum biogenesis and motility. Nucleic Acids Res 40:1107311085.

34. Spöring I, Felgner S, Preuße M, Eckweiler D, Rohde M, Häussler S, Weiss S, Erhardt M. 2018. Regulation of Flagellum Biosynthesis in Response to Cell Envelope Stress in Salmonella enterica Serovar Typhimurium. MBio 9. 
523 35. Laganenka L, López ME, Colin R, Sourjik V. 2020. Flagellum-Mediated Mechanosensing 524 and RfIP Control Motility State of Pathogenic Escherichia coli. MBio 11.

525 36. Martin RG, Rosner JL. 2001. The AraC transcriptional activators. Curr Opin Microbiol $526 \quad 4: 132-137$.

527 37. Wu J, Weiss B. 1992. Two-stage induction of the soxRS (superoxide response) regulon of $528 \quad$ Escherichia coli. J Bacteriol 174:3915-3920.

38. Nunoshiba T, Hidalgo E, Amábile Cuevas CF, Demple B. 1992. Two-stage control of an oxidative stress regulon: the Escherichia coli SoxR protein triggers redox-inducible expression of the soxS regulatory gene. J Bacteriol 174:6054-6060.

39. Griffith KL, Shah IM, E. Wolf R. 2004. Proteolytic degradation of Escherichia coli transcription activators SoxS and MarA as the mechanism for reversing the induction of the superoxide (SoxRS) and multiple antibiotic resistance (Mar) regulons. Mol Microbiol

40. Martin RG, Rosner JL. 2002. Genomics of the marA/soxS/rob regulon of Escherichia coli: identification of directly activated promoters by application of molecular genetics and informatics to microarray data. Mol Microbiol 44:1611-1624.

41. Seo SW, Kim D, Szubin R, Palsson BO. 2015. Genome-wide Reconstruction of OxyR and SoxRS Transcriptional Regulatory Networks under Oxidative Stress in Escherichia coli K183:3890-3902. 
43. Greenberg JT, Monach P, Chou JH, Josephy PD, Demple B. 1990. Positive control of a global antioxidant defense regulon activated by superoxide-generating agents in Escherichia coli. Proc Natl Acad Sci U S A 87:6181-6185.

44. Nunoshiba T, Demple B. 1994. A cluster of constitutive mutations affecting the C-terminus of the redox-sensitive SoxR transcriptional activator. Nucleic Acids Res 22:2958-2962.

45. Koirala S, Rao CV. 2017. Dynamic Measures of Flagellar Gene Expression. Methods Mol Biol 1593:73-83.

46. Wozniak CE, Lee C, Hughes KT. 2009. T-POP array identifies EcnR and Pefl-SrgD as novel regulators of flagellar gene expression. J Bacteriol 191:1498-1508.

47. Kühne C, Singer HM, Grabisch E, Codutti L, Carlomagno T, Scrima A, Erhardt M. 2016. RfIM mediates target specificity of the RcsCDB phosphorelay system for transcriptional repression of flagellar synthesis in Salmonella enterica. Mol Microbiol 101:841-855.

48. Zhou X, Meng X, Sun B. 2008. An EAL domain protein and cyclic AMP contribute to the interaction between the two quorum sensing systems in Escherichia coli. Cell Res 18:937948.

49. Gama-Castro S, Jiménez-Jacinto V, Peralta-Gil M, Santos-Zavaleta A, Peñaloza-Spinola MI, Contreras-Moreira B, Segura-Salazar J, Muñiz-Rascado L, Martínez-Flores I, Salgado H, Bonavides-Martínez C, Abreu-Goodger C, Rodríguez-Penagos C, Miranda-Ríos J, Morett E, Merino E, Huerta AM, Treviño-Quintanilla L, Collado-Vides J. 2008. RegulonDB (version 6.0): gene regulation model of Escherichia coli K-12 beyond transcription, active (experimental) annotated promoters and Textpresso navigation. Nucleic Acids Res 36:D120-4.

50. Bailey TL, Boden M, Buske FA, Frith M, Grant CE, Clementi L, Ren J, Li WW, Noble WS. 
2009. MEME SUITE: tools for motif discovery and searching. Nucleic Acids Res 37:W202-

8.

570 51. Hartog E, Ben-Shalom L, Shachar D, Matthews KR, Yaron S. 2008. Regulation of marA, 571 soxS, rob, acrAB and micF in Salmonella enterica serovar Typhimurium. Microbiol Immunol 52:565-574.

573 52. Martin RG, Gillette WK, Rhee S, Rosner JL. 1999. Structural requirements for marbox 574 function in transcriptional activation of mar/sox/rob regulon promoters in Escherichia coli: sequence, orientation and spatial relationship to the core promoter. Mol Microbiol 34:431441.

53. Wood TI, Griffith KL, Fawcett WP, Jair KW, Schneider TD, Wolf RE Jr. 1999. Interdependence of the position and orientation of SoxS binding sites in the transcriptional activation of the class I subset of Escherichia coli superoxide-inducible promoters. Mol Microbiol 34:414-430.

54. Sittka A, Pfeiffer V, Tedin K, Vogel J. 2007. The RNA chaperone Hfq is essential for the virulence of Salmonella typhimurium. Mol Microbiol 63:193-217.

55. Wang P, Zhang H, Liu Y, Lv R, Liu X, Song X, Wang J, Jiang L. 2020. SoxS is a positive regulator of key pathogenesis genes and promotes intracellular replication and virulence of Salmonella Typhimurium. Microb Pathog 139:103925.

56. Stewart MK, Cummings LA, Johnson ML, Berezow AB, Cookson BT. 2011. Regulation of phenotypic heterogeneity permits Salmonella evasion of the host caspase-1 inflammatory response. Proc Natl Acad Sci U S A 108:20742-20747. resistance of Salmonella typhimurium to paraquat but not for virulence in mice. Infect 
Immun 65:5371-5375.

592

593

594

595

596

597

598

599

600

58. Perez JC, Groisman EA. 2009. Evolution of transcriptional regulatory circuits in bacteria. Cell 138:233-244.

59. Ilyas B, Mulder DT, Little DJ, Elhenawy W, Banda MM, Pérez-Morales D, Tsai CN, Chau NYE, Bustamante VH, Coombes BK. 2018. Regulatory Evolution Drives Evasion of Host Inflammasomes by Salmonella Typhimurium. Cell Rep 25:825-832.e5.

60. Datsenko K a., Wanner BL. 2000. One-step inactivation of chromosomal genes in Escherichia coli K-12 using PCR products. Proc Natl Acad Sci U S A 97:6640-6645.

61. Thierauf A, Perez G, Maloy AS. 2009. Generalized transduction. Methods Mol Biol $501: 267-286$.

62. Saini S, Pearl J a., Rao CV. 2009. Role of FimW, FimY, and FimZ in regulating the expression of Type I fimbriae in Salmonella enterica serovar Typhimurium. J Bacteriol $191: 3003-3010$.

63. Ellermeier CD, Janakiraman A, Slauch JM. 2002. Construction of targeted single copy lac fusions using lambda Red and FLP-mediated site-specific recombination in bacteria. Gene 290:153-161.

64. Rappleye CA, Roth JR. 1997. A Tn10 derivative (T-POP) for isolation of insertions with conditional (tetracycline-dependent) phenotypes. J Bacteriol 179:5827-5834.

65. Lee C, Wozniak C, Karlinsey JE, Hughes KT. 2007. Genomic screening for regulatory genes using the T-POP transposon. Methods Enzymol 421:159-167.

66. Slauch JM, Silhavy TJ. 1991. cis-acting ompF mutations that result in OmpR-dependent constitutive expression. J Bacteriol 173:4039-4048. 
bioRxiv preprint doi: https://doi.org/10.1101/2020.05.29.124750; this version posted May 31, 2020. The copyright holder for this preprint (which was not certified by peer review) is the author/funder. All rights reserved. No reuse allowed without permission.

613 67. Edgar RC. 2004. MUSCLE: multiple sequence alignment with high accuracy and high 614 throughput. Nucleic Acids Res 32:1792-1797. 


\section{FIGURES LEGENDS}

2 Figure 1. SoxS-dependent repression of flagellar genes and motility occurs after Class I

3 transcription. A) Levels of of flhDC (LCM2324, LCM2471), flhB (LCM2325, LCM2472) and fliC

4 (LCM2326, LCM2473) transcription in sox $R^{\text {Con }}$ and WT background strains, respectively. Data

5 are presented as the percentage of the wild-type expression level for each class of flagellar

6 promoter. $\left({ }^{*}\right)$ indicates Student's t-test $P \leq 10^{-6}$. B) The effect of constitutive expression of SoxS

$7 \quad\left(\right.$ sox $\left.R^{\mathrm{Con}}\right)$ on FlhC and FliC protein levels. Western blots were conducted with $100 \mu \mathrm{g}$ total

8 protein. Anti-FLAG antibody was used to detect FlhC-3XFLAG expressed in sox $R^{\text {Con }}$ (LCM2697)

9 and $\operatorname{sox} R^{\mathrm{WT}}$ (LCM2696) background strains, respectively. Anti-FliC antibody was used to detect

10 the flagellin protein, FliC in $\operatorname{sox} R^{\mathrm{Con}}(\mathrm{LCM} 2449)$ and sox $R^{\mathrm{WT}}$ (LCM1930) background strains,

11 respectively. The loading control DnaK was detected by using anti-DnaK antibody. C)

12 Swimming motility in sox $R^{\mathrm{WT}}$ (LCM1930) and sox $R^{\mathrm{Con}}$ (LCM2449) background strains,

13 respectively.

15 Figure 2. Inactivation of rfIP alleviates SoxS-dependent flagellar repression. A) The relative 16 location of T-POP insertion sites in the rflP gene. Insertions are indicated by red arrows. T-POP 17 insertions in rflP were identified at genome coordinates $1,422,579 ; 1,423,247 ; 1,423,428$;

$181,423,238 ; 1,423,248$; and 1,423,344bp (ordered from left to right). B) Expression of flhB (Class 19 II) and fliC (Class III) genes in WT (LCM2325, LCM2326), sox $R^{\text {Con }}$ (LCM2472, LCM2473), $\Delta r f I P$ 20 (LCM2808, LCM2761) and soxR $R^{\text {Con } / \triangle r f I P ~(L C M 2809, ~ L C M 2762) ~ b a c k g r o u n d ~ s t r a i n s, ~ r e s p e c t i v e l y . ~}$ 21 Data are presented as the percentage of the wild-type expression level for each class of 22 flagellar promoter. $\left({ }^{*},{ }^{* *}\right)$ indicates a Student's $t$-test $P \leq 0.002$ or $P \leq 10^{-6}$. C) FlhC-3XFLAG 23 protein levels in WT (LCM2764), sox $R^{\mathrm{Con}}$ (LCM2765), $\Delta r f I P\left(\right.$ LCM2766) and sox $R^{\mathrm{Con}} / \Delta r f I P$

24 (LCM2767) background strains. Anti-FLAG antibody was used to detect FlhC-3XFLAG and anti25 DnaK antibody was used to detect the loading control, DnaK. D) Swimming motility observed in 


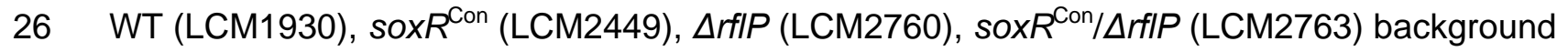
27 strains.

29 Figure 3. Complementation of SoxS-dependent flagellar gene repression in sox $R^{\text {Con }} / \Delta r f I P$ strain. A) Levels of fliC expression in sox $R^{\text {Con }}$ (LCM2473) and sox $R^{\text {Con }} / \Delta r f I P$ (LCM2762) background

31 strains with pWKS30 (empty vector) or pRfIP (rflP complementation vector). Data are presented 32 as the percentage of the fliC expression level in the sox $R^{\text {Con }}$ background harboring pWKS30. $\left({ }^{*}\right)$

33 indicates a Student's $t$-test $P \leq 10^{-5}$. B) Swimming motility in soxR ${ }^{\text {Con }}$ (LCM2449) and

34 soxRCon/ArflP (LCM2763) strains with vectors pWKS30 and pRflP.

Figure 4. The effects of SoxS on rfIP transcription. A) Levels of $r f P^{\prime}$-lacZY activity in wild-type

37 (LCM2791) and sox $R^{\text {Con }}$ (LCM2792) genetic backgrounds. Data are presented as a fold increase in $\beta$-galactosidase activity compared to wild-type (LCM2791). Comparison of wild-type

39 and $\operatorname{sox} R^{\mathrm{Con}}$ with the Student's $t$-test resulted in a $P=4.8 \times 10^{-6}$. B) The promoter region of $r f I P$

$40(1,422,425$ to $1,422,575)$. The putative SoxS binding motif (soxbox) identified by MEME analysis

41 is highlighted in blue. The guanine nucleotide highlighted in red is the transcription start site 42 (TSS) of the rfIP mRNA identified from 5'-RACE in wild-type. 


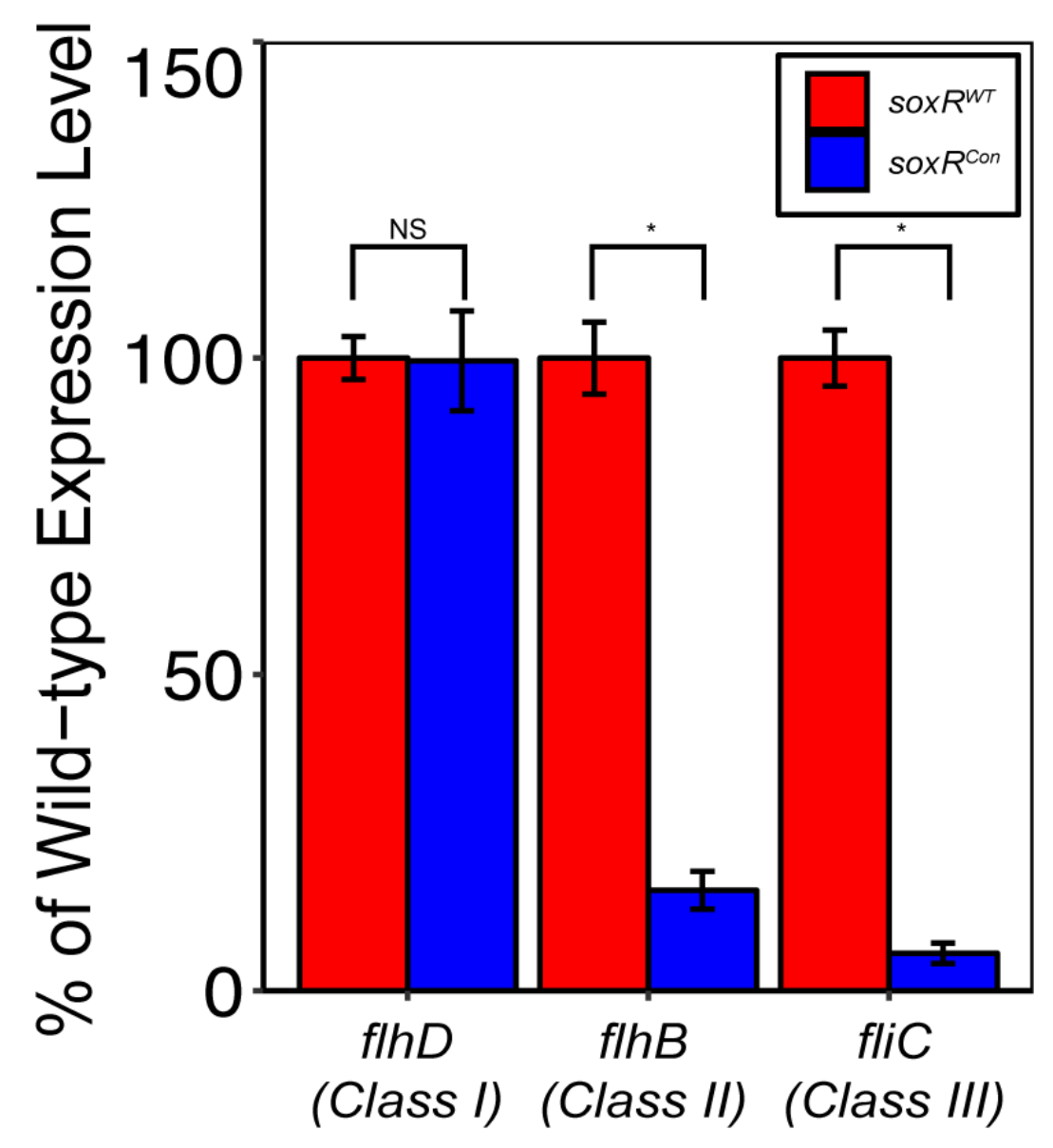

Promoter fusion

(Class I) (Class II) (Class III)

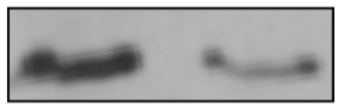

$\alpha-F L A G(F I h C)$

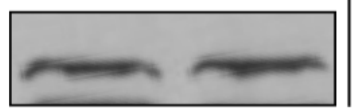
a-DnaK

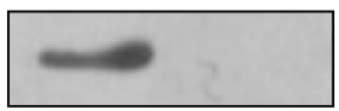
a-FliC

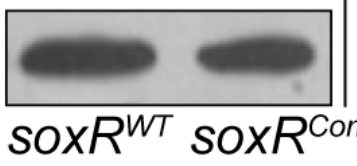
a-DnaK
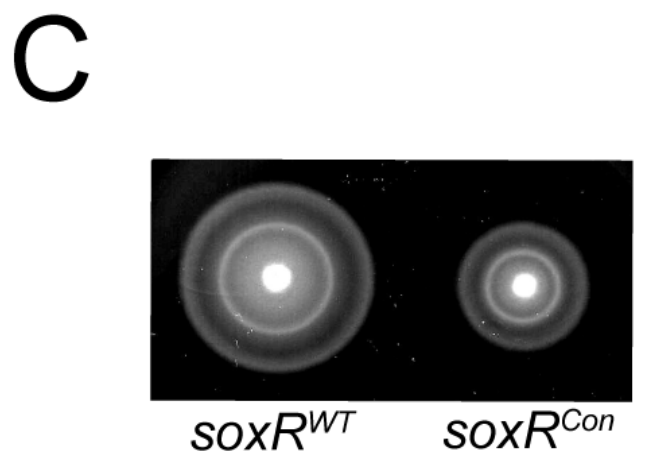

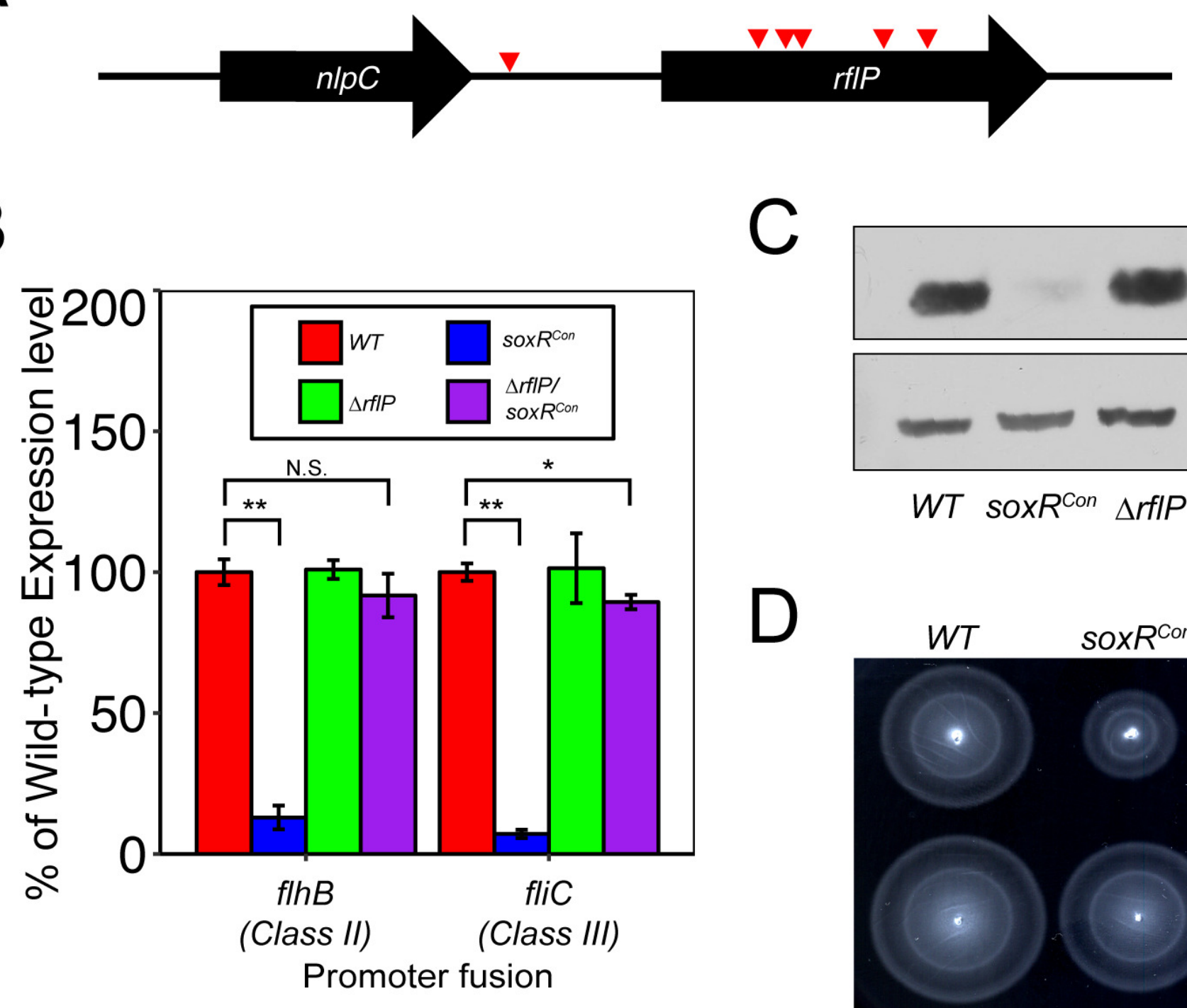
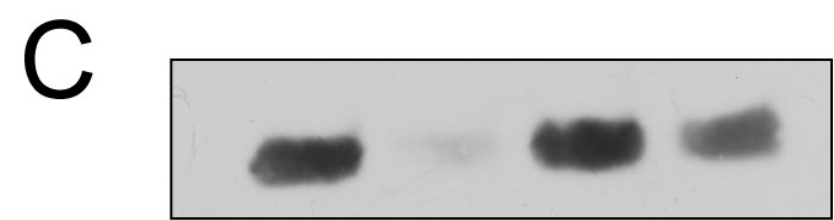

a-FLAG

$(\mathrm{FlhC})$

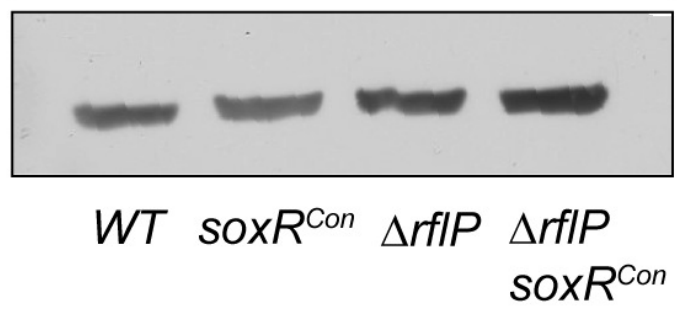

$\alpha-D n a K$

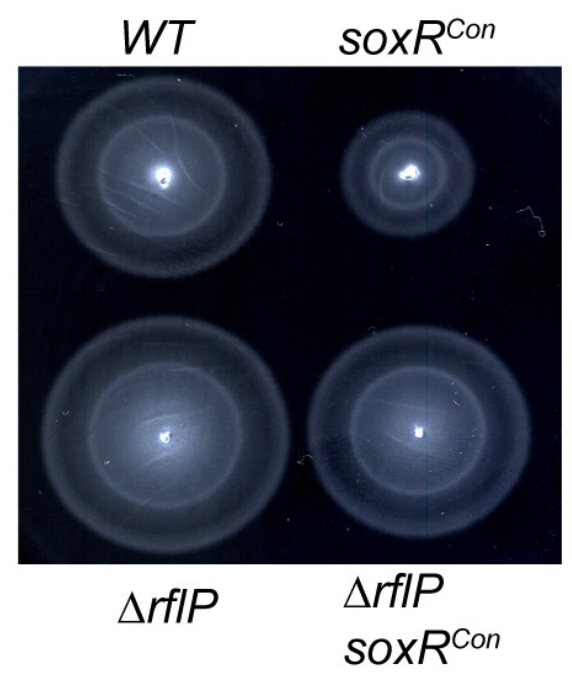




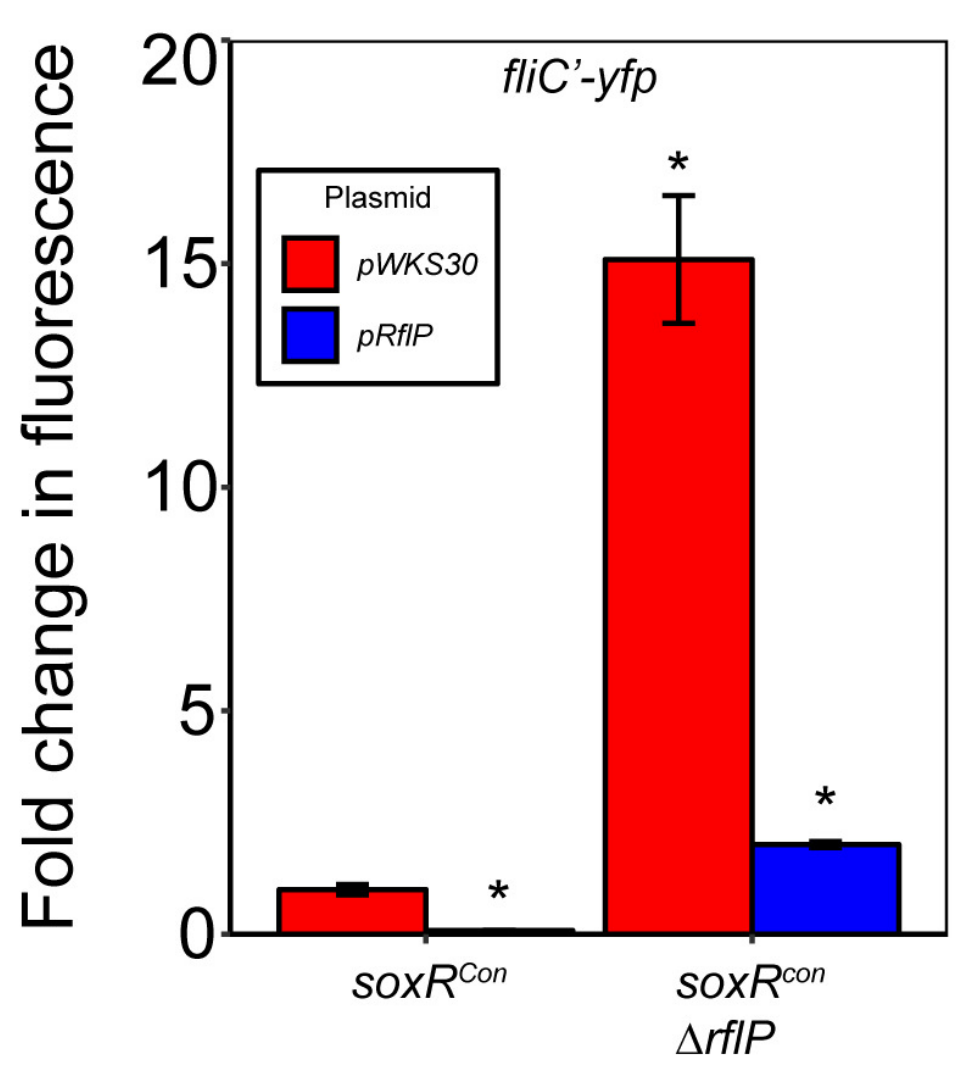

B

Plasmid

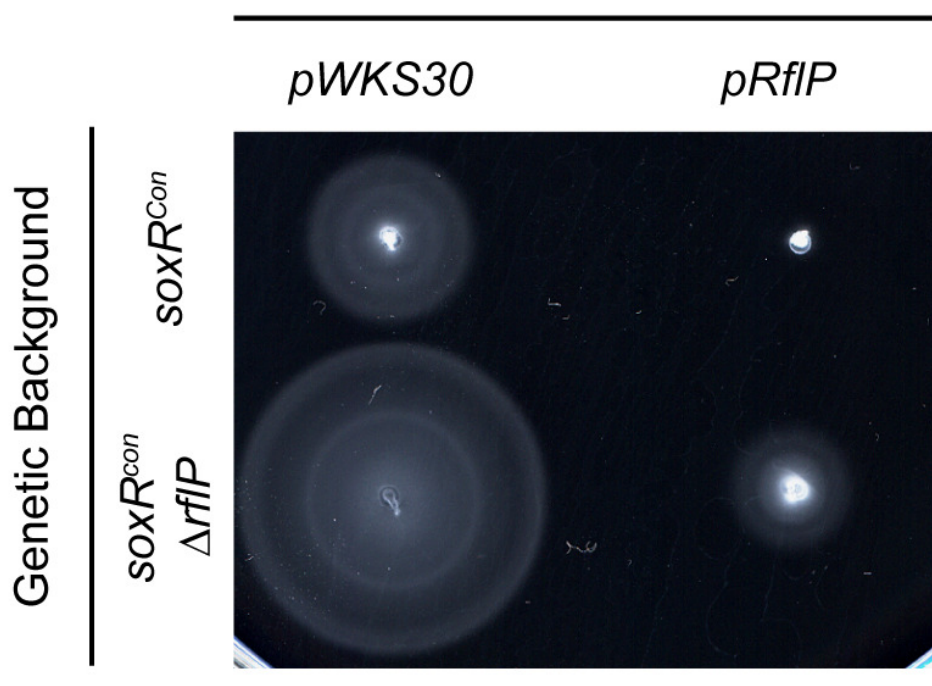


A

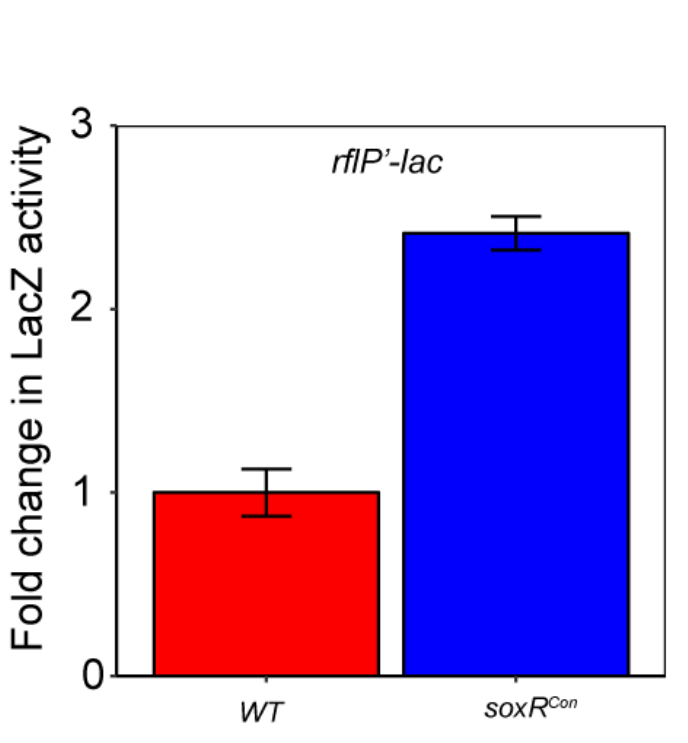

B

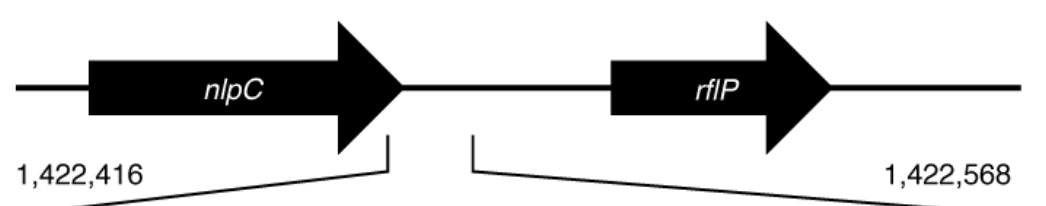

S. enterica - - C G T T C A T C G C T G G A T A A T G T G T A C T G G C E. coli T G C G T T C C T C A C T T G A T A A T G T C T A T T G G C

G A A A A A A C T T C T G G C A G G C A C G G C G A A T T T A G A A A A T T T C T G G C A G G C G A G A C GA A T C T A A C C T - . - . - . C G C A G A C G A T A T A G T A T G T C G A A A A $\mathrm{T}$ T G A C G G C G A T A T A C * * * * * * ****

А А А Т Т Т А Т А A --1 T C G C C G T C C A T T T A C T C C T G A A T A C
$*$ - T TA A C A TA TCG C C G G T T A A C T TA T G A C A

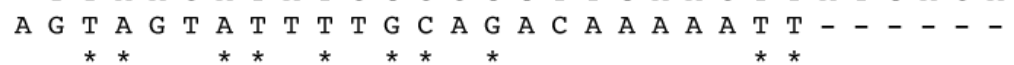
T C T G T T G T C A T C A T A T A T A A T G A T A T A C C - 